\title{
W/Z+Jet Cross Section Measurements at the Large Hadron Collider
}

\author{
Jason A. Nielsen \\ on behalf of the ATLAS and CMS collaborations \\ Santa Cruz Institute for Particle Physics \\ University of California, Santa Cruz, CA 95064, USA
}

\begin{abstract}
The Large Hadron Collider will open a new regime for the study of jet production in association with $\mathrm{W}$ and $\mathrm{Z}$ bosons. Cross section measurements developed by the ATLAS and CMS collaborations will probe perturbative quantum chromodynamics, and they will also help constrain $\mathrm{W} / \mathrm{Z}+$ jet contributions as background to new physics. The measurements are corrected for major experimental effects to facilitate comparison with theoretical calculations.
\end{abstract}

The study of jet production in association with $\mathrm{W}$ and $\mathrm{Z}$ bosons has advanced significantly during Run II of the Fermilab Tevatron, and we are poised to learn even more about these processes in high-energy proton-proton collisions at the CERN Large Hadron Collider $[1,2]$. Indeed, $\mathrm{W} / \mathrm{Z}+$ jet production (sometimes collectively labeled "V+jets") is enhanced in proton-proton LHC collisions relative to proton-antiproton Tevatron collisions because of the additional quark-quark interactions and the larger available phase space. Measurements of $\mathrm{W} / \mathrm{Z}+$ jet production at the $\mathrm{LHC}$ will probe unexplored regions of perturbative QCD, offer a testbed for calibrating detector response and efficiencies, and constrain backgrounds for new physics searches.

Production of $\mathrm{W}$ and $\mathrm{Z}$ bosons in association with jets is a high- $Q^{2}$ process, but at LHC energies of 10 to $14 \mathrm{TeV}$ even low- $x$ partons contribute to the cross section. Inclusive $\mathrm{W}$ and $\mathrm{Z}$ results may be more sensitive probes of PDFs at slightly lower $Q^{2}$ values, but the LHC offers a unique opportunity to test evolution with large cross sections in the region of high $Q^{2}$. The reach to low- $x$ regimes makes it possible to study the parton distribution functions of sea quarks as well as the ratio of up and down quark densities.

The well-known properties of the $\mathrm{W}$ and $\mathrm{Z}$ bosons serve as calibration parameters in $\mathrm{W} / \mathrm{Z}+$ jet events. Such events can be used to calculate lepton reconstruction and trigger efficiencies. The jets in the $\mathrm{Z}+$ jet events can be balanced against the fully reconstructed $\mathrm{Z}$ boson to derive jet energy calibrations, and the $\mathrm{W}$ transverse mass distribution can be compared with the theoretical lineshape to validate missing transverse energy measurements.

\section{Jet production in association with bosons}

The ATLAS and CMS collaborations have developed preliminary studies of cross section measurements at center-of-mass energy $\sqrt{s}=14 \mathrm{TeV}[3,4,5,6]$. These studies use a combination of leading-order matrix element calculations and parton showering codes to generate signal event samples. Particular care is taken to avoid double-counting of the phase space in the matrix element and parton shower. Special jet matching studies are required for the $\mathrm{W} / \mathrm{Z}$ generation in association with heavy flavor jets ( $\mathrm{b}$ and $\mathrm{c}$ quark jets).

One key to the cross section measurements in $\mathrm{W} / \mathrm{Z}+$ jet events is a reliable estimate of the trigger efficiency. Since the $\mathrm{Z}$ boson decays to two same-flavour leptons, it is possible to accept a trigger that fired on one lepton and test the same trigger hypothesis on the 
second lepton. The single lepton trigger efficiency can be measured as a function of the jet multiplicity with this tag-and-probe method (Fig. 1).

Here, the hadronic activity from the jets reduces the efficiency of the trigger isolation requirement for the lepton. The result is used to correct the measured cross section and to adjust the expected background to new physics searches from other lepton + jets events.

Typical requirements for $\mathrm{W}$ reconstruction in ATLAS are a single identified electron or muon with $p_{T}>20 \mathrm{GeV} / c$ and missing transverse energy $>25 \mathrm{GeV}$. The analyses presented here use a standard conebased jet clustering algorithm with a characteristic radius $\Delta R=0.4$. Figure 2 shows the number of jets produced in association with a reconstructed $\mathrm{W} \rightarrow \mu \nu$ decay. The contribution from true $\mathrm{W}+$ jet production

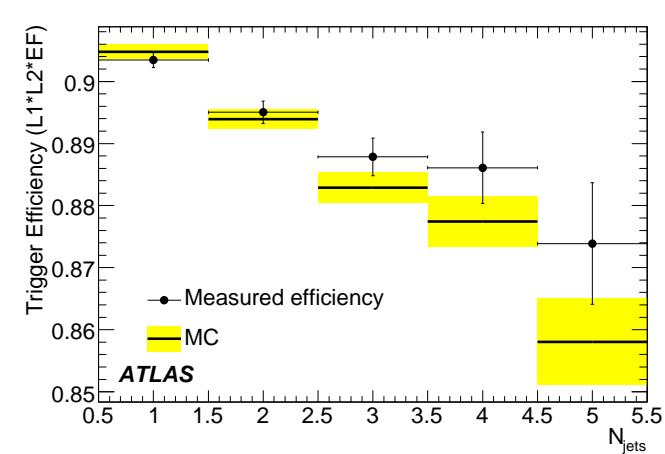

Figure 1: Simulated measurement of the electron trigger efficiency using the tag-and-probe method. is shown together with contributions from other Standard Model processes that form the background. Studies with other jet clustering algorithms are underway.

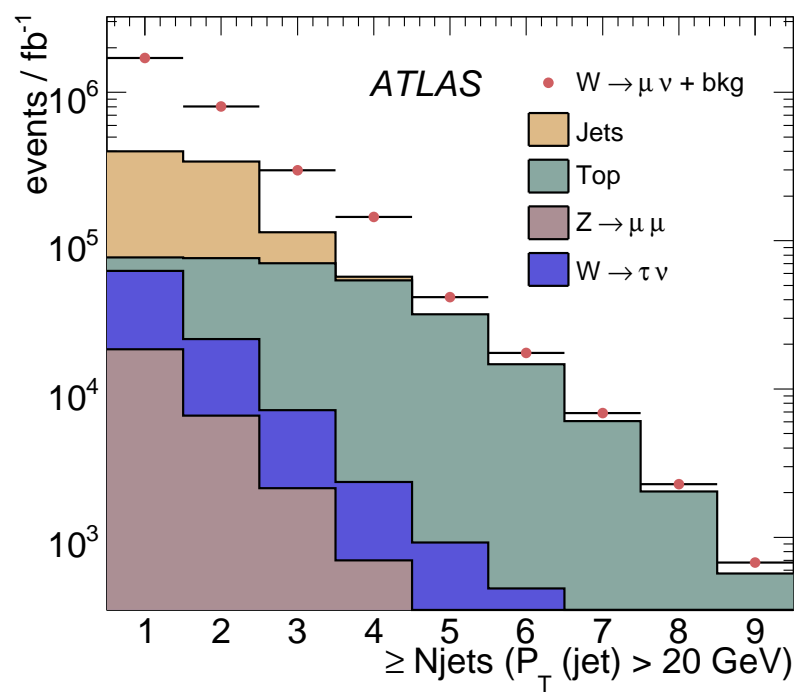

Figure 2: Expected inclusive jet multiplicity distribution in events selected by the $\mathrm{W} \rightarrow \mu \nu$ analysis. 
Typical requirements for $\mathrm{Z}$ reconstruction are two identified same-flavor oppositesign electrons $\left(E_{T}>25 \mathrm{GeV}\right)$ or muons $\left(p_{T}>15 \mathrm{GeV} / c\right)$. An isolated di-electron or single electron trigger is used for the electron channel, while an isolated di-muon trigger is used for the muon channel. The CMS $\mathrm{Z}+\mathrm{b} \overline{\mathrm{b}}$ measurement reconstructs a $\mathrm{Z} \rightarrow \ell^{+} \ell^{-}$signature in addition to jets clustered with $\Delta R=0.5$. At least two jets are required to pass a b-tagging selection based on the three-dimensional track impact parameter significance. Tracks from heavy flavor jets are more likely to have a significantly non-zero IP significance than tracks from light flavor jets because of the long lifetime and large mass of the $\mathrm{b}$ and c quarks. The dilepton invariant mass distribution expected with an integrated luminosity of $100 \mathrm{pb}^{-1}$ is shown in Fig. 3. The early measurement of $\mathrm{Z}+\mathrm{b} \overline{\mathrm{b}}$ will constrain an important background for Higgs searches

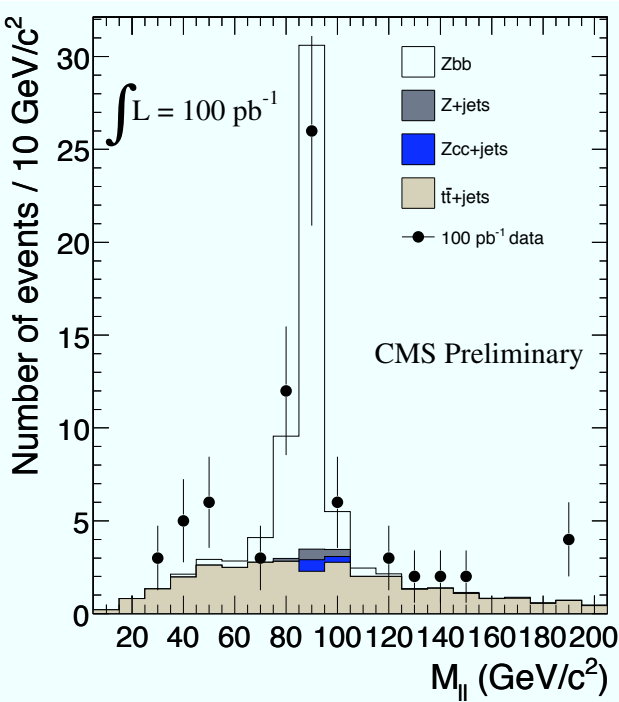

Figure 3: Expected dilepton invariant mass distribution for events with at least $2 \mathrm{~b}$-tagged jets. in the $\mathrm{ZH}$ channel.

All of these preliminary studies estimate background contributions from large samples of simulated Standard Model events. Further studies are now focusing on methods of estimating background from collected data samples instead of simulated events. Most methods define control regions with little $\mathrm{W} / \mathrm{Z}+$ jets signal and extrapolate to the signal region of isolated leptons and large missing transverse energy. The enormous rate for $\mathrm{W}$ and $\mathrm{Z}$ events should make such methods robust and reliable.

\section{Selected studies of systematic uncertainties}

The systematic uncertainty on absolute cross section measurements is bounded by the luminosity measurement uncertainty. Current conservative estimates of the initial luminosity uncertainty range from $5 \%$ to $10 \%$ for ATLAS and CMS; these uncertainties will decrease as the dedicated luminosity monitors are commissioned. Measurements of the ratio $(\mathrm{W}+\mathrm{jets}) /(\mathrm{Z}+\mathrm{jets})$ and of the incremental jet rate are insensitive to the luminosity measurement and enjoy decreased uncertainty.

Other significant uncertainties on all W/Z+jet measurements include PDF uncertainties and jet energy scale uncertainties, both of which affect the signal efficiencies directly. The PDF uncertainties are calculated by reweighting the simulated events to match the CTEQ6M error sets [7]. Because jets are identified only within certain $\eta$ and $p_{T}$ ranges, a change in the PDFs modifies the jet distribution (see Fig. 4). Concurrent gluon PDF measurements with inclusive $\mathrm{W}$ events will reduce the uncertainties in all $\mathrm{W} / \mathrm{Z}+$ jet channels.

Even after careful detector calibration has been applied, some residual jet energy scale (JES) uncertainty arises from the absolute energy correction, underlying event correction, and energy outside the reconstructed jet. The JES uncertainty can result in a shift of the 

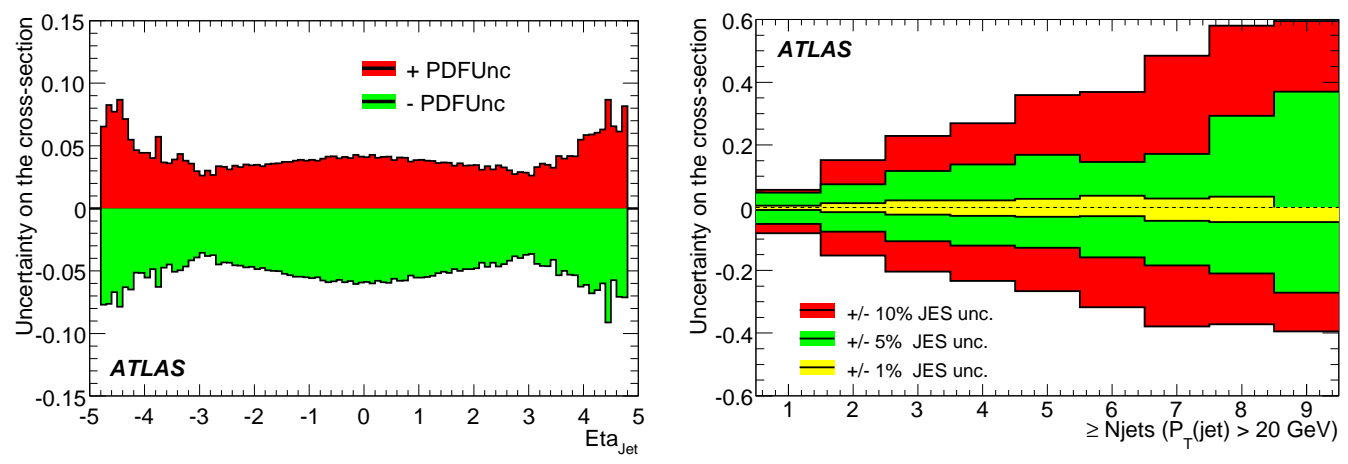

Figure 4: On left, relative uncertainty on the jet $\eta$ distribution from $\mathrm{W} \rightarrow \mathrm{e} \nu$ events due to PDF uncertainties. The increased uncertainty in the jet $\eta$ distribution at high $\eta$ corresponds to contributions from high- $Q^{2}$ low- $x$ PDFs. On right, relative uncertainty on the $\mathrm{W} \rightarrow$ $\mu \nu$ differential cross section measurement for different projected levels of jet energy scale uncertainty.

jet multiplicity spectrum, one that is reflected in the $\mathrm{W} / \mathrm{Z}+$ jet cross section measurements. Figure 4 shows the effect of the shifting the jet energies up and down by the full JES uncertainty for three possible values. It is clear that the effect due to JES is significant (and greater than the effect of the luminosity uncertainty) if the relative JES value is $5 \%$ or greater. The jet energy scale can be measured using $\mathrm{Z}+$ jet events because the $\mathrm{Z}$ decay to leptons, which is fully reconstructed, gives an estimate of the true momentum of the recoiling jet.

\section{Unfolding and correction techniques}

Past measurements of cross sections and boson $p_{T}$ distributions have been difficult to compare directly with theoretical calculations because the measurements were not corrected for detector effects. With the recent advances in next-to-leading order calculations of $\mathrm{W} / \mathrm{Z}+$ jet production, it is more important than ever to correct results to the particle (hadron) level for comparison with theory. Figure 5 shows the effects of a correction for the electron reconstruction and trigger efficiency, followed by a correction for the jet reconstruction efficiency and unfolding of the jet energy resolution. The result of this unfolding is directly comparable to jets formed from generator-level hadrons, after showering but before any detector interaction.

It is also possible to derive further corrections from the hadron level to the parton level, corresponding to the outgoing parton in matrix element calculations. Such correction functions can then be applied in reverse to make parton-level NLO Monte Carlo calculations comparable to hadron-level experimental measurements. The contribution of underlying event (UE) tends to increase jet energy relative to parton energy, most significantly for low energy jets. Parton fragmentation tends to decrease jet energy, as fragmentation products fall outside the reconstructed jet area. The two competing effects balance roughly for jets with $p_{T}>40 \mathrm{GeV} / c$, as shown in Fig. 5. This factor is estimated by switching off underlying

DIS 2009 
event and fragmentation in the showering program.
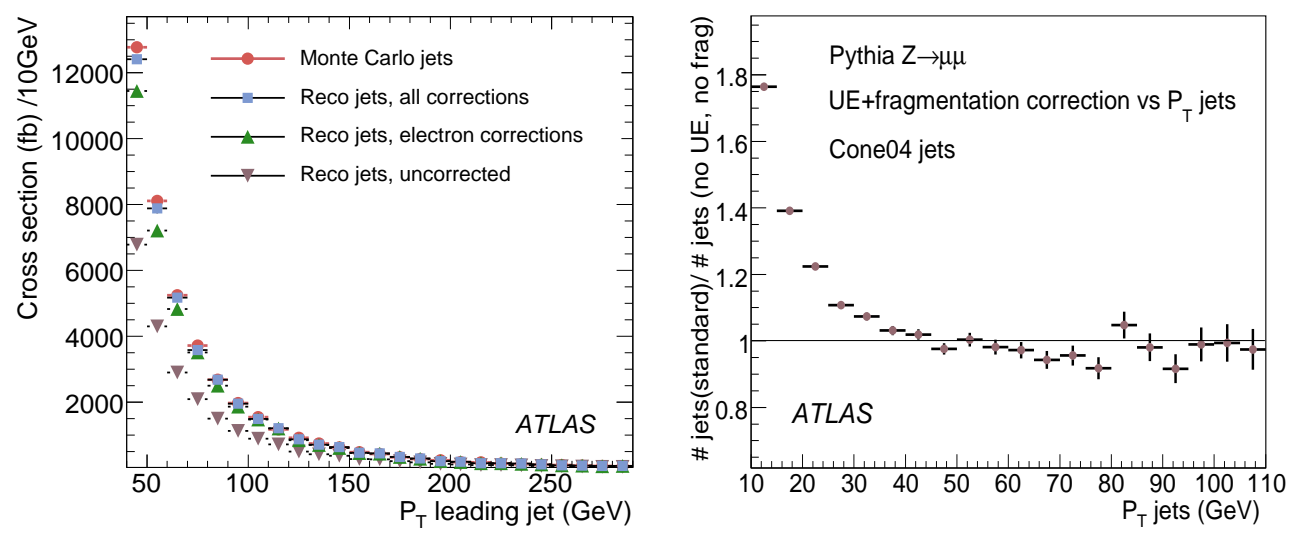

Figure 5: At left, effect of successive corrections to the jet distribution reconstructed in simulated $\mathrm{Z} \rightarrow \mathrm{e}^{+} \mathrm{e}^{-}$events. At right, the jet correction factor from hadron level to parton level in simulated $\mathrm{Z} \rightarrow \mu^{+} \mu^{-}$events.

\section{Conclusions}

Measurements of $\mathrm{W} / \mathrm{Z}+$ jet events in ATLAS and CMS benefit from high cross sections at the LHC while probing new QCD regimes. Preliminary studies have identified major sources of background and sources of systematic uncertainty in the main cross section measurements. Comparisons to theoretical calculations at the next-to-leading order level are facilitated by experimental unfolding techniques, which allow comparisons at particle (hadron) level. If the total uncertainty on the cross section measurement is of order $10 \%-20 \%$, the experimental measurements could distinguish between leading event generator models. Certain cross section ratios measurements, such as $\sigma(\mathrm{W}+\mathrm{jets}) / \sigma(\mathrm{Z}+\mathrm{jets})$, are being pursued as a way to reduce the systematic uncertainties. The measurements of $\mathrm{W} / \mathrm{Z}+$ jet production cross sections and jet multiplicity distributions will help constrain such processes and backgrounds for new physics searches at the LHC.

\section{References}

[1] T. Aaltonen et al. [CDF Collaboration], Phys. Rev. Lett. 100, 102001 (2008), T. Aaltonen et al. [CDF Collaboration], Phys. Rev. D 77, 011108 (2008).

[2] V. M. Abazov et al. [D0 Collaboration], Phys. Lett. B 669, 278 (2008).

[3] G. Aad et al. [The ATLAS collaboration], JINST 3:S08003, (2008).

[4] S. Chatrchyan et al. [The CMS collaboration], JINST 33:S08004 (2008).

[5] G. Aad et al. [The ATLAS Collaboration], "Expected Performance of the ATLAS Experiment - Detector, Trigger and Physics", CERN-OPEN-2008-020, arXiv:0901.0512.

[6] The CMS Collaboration, "Measurement of the $\mathrm{Zb}^{+} \mathrm{b}^{-}, \mathrm{Z} \rightarrow \ell^{+} \ell^{-}$cross section with $100 \mathrm{pb}^{-1}$ of early CMS data at the LHC," CMS PAS EWK-08-001.

[7] J. Pumplin, D. R. Stump, J. Huston, H. L. Lai, P. M. Nadolsky and W. K. Tung, global QCD JHEP 0207, 012 (2002) [arXiv:hep-ph/0201195]. 